\title{
Sarcoidosis Associated Pulmonary Hypertension
}

\author{
Veronica Palmero, Phillip Factor and Roxana Sulica \\ Albert Einstein College of Medicine, Beth Israel Medical Center, New York \\ United States of America
}

\section{Introduction}

Pulmonary hypertension (PH) is a serious complication of sarcoidosis, a multi-systemic inflammatory disease characterized by the presence of widespread non-caseating granulomas. When PH develops in patients with sarcoidosis, it is associated with increased morbidity and mortality.

Sarcoidosis-associated pulmonary hypertension (SAPH) is most commonly seen in patients with advanced pulmonary sarcoidosis as a result of pulmonary fibrosis and chronic hypoxemia. However, the presence of $\mathrm{PH}$ in individuals with extra-pulmonary sarcoidosis and normal pulmonary physiology suggests that SAPH may be due to other mechanisms. Appropriate understanding of this disease and its complex pathophysiology is essential for early recognition and therapy.

\section{Definition}

SAPH is defined as mean pulmonary artery pressure (mPAP) above $25 \mathrm{~mm} \mathrm{Hg}$ at rest with a pulmonary artery occlusion pressure (PAOP) or left ventricular end-diastolic pressure (LVEDP) less than $15 \mathrm{mmHg}$, in patients with sarcoidosis, diagnosed by clinical, radiological and histological criteria, in the absence of other risk factors for pulmonary hypertension. (Badesch et al., 2009) Because of the complex pathogenetic mechanisms of SAPH, it is classified under group 5 (i.e. Pulmonary Hypertension with Unclear Multifactorial Mechanisms) of the Updated Clinical Classification of Pulmonary Hypertension (Dana Point, 2008). (Simonneau et al., 2009)

\section{Epidemiology}

The prevalence of SAPH in general sarcoidosis population is $5 \%$ to $28 \%$ and varies with geographical location, clinical characteristics and diagnostic method. (Palmero \& Sulica, 2010)

In case series of unselected sarcoidosis patients, the prevalence of SAPH is as low as $5-15 \%$. If only dyspneic patients are considered, SAPH frequency can be as high as $60 \%$. (Baughman et al., 2011) The prevalence of SAPH has been reported to be $73.8 \%$ in patients being evaluated for lung transplantation, with severe $\mathrm{PH}(\mathrm{mPAP}>40 \mathrm{~mm} \mathrm{Hg}$ ) present in $36 \%$ of patients. (Shorr et al., 2005) SAPH is more prevalent in patients with advanced radiographic stage, but can develop in the absence of parenchymal lung abnormalities. In one cohort of 54 patients with sarcoidosis and elevated pulmonary pressures by 
echocardiography, $60 \%$ of the patients had radiographic stage IV disease, while $10 \%$ had radiologically normal lung parenchyma (stages 0 and I). (Sulica et al., 2005) When right heart catheterization (RHC) was used to measure mPAP, pulmonary hypertension was found in $31.8 \%$ of 22 patients without lung fibrosis. (Nunes et al., 2006)

\section{Pathophysiology}

Multiple mechanisms have been proposed to explain the development of pulmonary hypertension in patients with sarcoidosis. Pulmonary fibrosis with destruction of the pulmonary vascular bed and chronic hypoxemia is a major cause of increased pulmonary artery pressures in advanced pulmonary sarcoidosis. Since SAPH may develop in the absence of pulmonary fibrosis, alternative pathogenic mechanisms have been proposed and include elevated levels of endothelin-1 (ET-1), decreased nitric oxide synthesis, structural abnormalities such as granulomatous vasculitis, pulmonary veno-occlusive disease, external compression of the pulmonary vessels by enlarged mediastinal lymph nodes, hypoxiainduced vasoconstriction, and pulmonary vascular remodeling. In addition, a significant percent (i.e. approximately $30 \%$ ) of patients with sarcoidosis have elevated pulmonary artery pressures due to left heart disease. (Baughman et al., 2010).

Presence of multiple mechanisms of increased pulmonary pressures in sarcoidosis underscores the importance of different management and therapeutic options in SAPH.

\subsection{Nitric oxide}

The synthesis and release of nitric oxide (NO) from the endothelial cells and other sources is a key element in the maintenance of the low pulmonary artery vasomotor tone characteristic of the normal pulmonary circulation. (Sterling \& Creager, 1999) Decreased production of NO has been associated with the development of various forms of pulmonary hypertension. There is indirect evidence that a NO-dependent mechanism is operative in certain cases of $\mathrm{SAPH}$. Inhaled NO administration acutely reduces the pulmonary vascular resistance (PVR) up to $20 \%$ in patients with SAPH, even in the presence of significant parenchymal lung disease. Although PVR reduction was not maintained over time, specific treatment targeting NO production might still be beneficial. (Preston et al., 2001)

\subsection{Endothelin-1}

Endothelin-1 (ET-1) is a peptide synthesized by the vascular endothelium with potent vasoconstrictor, pro-inflammatory and proliferative properties. This peptide has been found to contribute to increased pulmonary arterial tone and smooth-muscle proliferation in patients with pulmonary arterial hypertension. (Giaid et al., 1993) Similarly, elevated levels of ET-1 in plasma, bronchio-alveolar lavage and urine have been found in SAPH. Moreover, ET-1 levels decrease with clinical remission of sarcoidosis. (Letizia et al, 2001; Reichenberger et al., 2001; Sofia et al., 1995; Terashita et al., 2006) These findings suggest an important role for ET-1 in the pathogenesis of sarcoidosis and $\mathrm{PH}$ and raise the possibility of use of endothelin receptor antagonists in patients with SAPH.

\subsection{Granulomatous vasculitis}

Sarcoidosis is characterized by the presence of generalized granulomatous inflammation in organs and tissues, including the pulmonary vascular wall. This includes inflammation and 
necrosis of the vessel wall with destruction of the elastic media of small and medium-sized vessels, with subsequent vascular remodeling and occlusive narrowing of the vascular lumen. These changes are reflected hemodynamically in elevated pulmonary vascular resistance and pulmonary hypertension. (Takemura et al., 1991, 1992)

Granulomatous angiitis has been found in $69-100 \%$ of lung biopsies from patients with sarcoidosis, with a predilection for venous structures, although arterial involvement has been reported as well. (Rosen et al., 1977)

\subsection{Pulmonary veno-occlusive disease (PVOD)}

PVOD is an occlusive venopathy characterized by extensive and diffuse fibrosis of the intima of the venules and small intralobular veins. Recanalization occurs over time, resulting in chronic hemosiderosis and calcium deposition in the elastic laminae, altering the normal hemodynamics. These changes have been found in explanted lung specimens of patients with sarcoidosis, suggesting an association of a PVOD-like disease with the development of pulmonary hypertension in sarcoidosis. (Nunes et al., 2006) Typical radiologic findings of PVOD, such as ground glass opacities and inter-lobular septal edema, have been frequently found in patients with SAPH, suggesting that PVOD accounts for some of the PVR increase seen in SAPH. (Handa et al., 2006; Nunes et al., 2006)

\subsection{Extrinsic compression of pulmonary vasculature}

Extrinsic compression of the pulmonary vasculature by enlarged lymph nodes may be associated with significant increases in PVR and mPAP if the vascular lumen is reduced more than $50 \%$. Nunes et al. described extrinsic compression of large pulmonary arteries by lymphadenopathy in $21.4 \%$ of patients with sarcoidosis that developed pulmonary hypertension. It was typically attributed to bilateral mediastinal and hilar lymph node involvement. Sarcoidosis-associated mediastinal fibrosis can also contribute to external compression of the pulmonary vasculature. The diagnostic method of choice in cases of extrinsic pulmonary artery compression is pulmonary angiography, which demonstrates extrinsic pulmonary artery stenosis without intraluminal obstruction. (Damuth et al., 1980; Nunes et al., 2006; Wescott \& DeGraff, 1973)

\subsection{Hypoxia-induced vasoconstriction}

Chronic hypoxemia and resulting pulmonary vascular remodeling are implicated in the pathogenesis of pulmonary hypertension in chronic parenchymal lung disease. Correction of hypoxemia with supplemental oxygen has been shown to ameliorate the progression of pulmonary hypertension in patients with COPD. It is reasonable to extrapolate that patients with sarcoidosis and impaired pulmonary function, destruction of the lung parenchyma and hypoxemia are at similar risk for pulmonary hypertension by the same mechanisms.

\subsection{Pulmonary hypertension due to left heart disease}

Cardiac factors, either direct granulomatous myocardial involvement or left ventricular diastolic dysfunction with impaired relaxation have also been linked with increased pulmonary artery pressures in patients with sarcoidosis. In these circumstances, pulmonary hypertension is postcapillary in nature (i.e. pulmonary venous hypertension). This 
mechanism implies a different prognosis and requires different management strategies from precapillary SAPH treatment. (Baughman et al., 2006, 2010)

\section{Clinical presentation}

Persistent or progressive dyspnea on exertion in excess to that expected from the parenchymal lung involvement is the sentinel symptom of SAPH. (Handa et al., 2006; Nunes et al., 2006; Sulica et al., 2005) Similarly, appearance or worsening of dyspnea in a patient with stable or improved pulmonary function or despite optimization of immunosuppressive therapy may suggest SAPH. (Baughman et al., 2010) Other symptoms such as cough, chest pain, palpitations, and pre-syncope or syncope are suggestive, but not specific of SAPH. Less than $10 \%$ of patients with SAPH are asymptomatic. SAPH should be considered in patients presenting with syncope, although this symptom could also represent sarcoid cardiac involvement. (Sulica et al., 2005) Specific signs of pulmonary hypertension, such as a loud pulmonary component of the second heart sound, tricuspid or pulmonary regurgitant murmurs, and right-sided gallop may be present in SAPH. Signs of right ventricular failure, like lower extremity edema, right ventricular heave, and elevated jugular venous pressure, are present in $21 \%$ of the patients with SAPH. (Sulica et al., 2005) Hypoxemia and decreased functional status, measured by the 6-minute-walk distance, exercise desaturation and decreased distance-saturation product (DSP), tend to be more pronounced in patients with SAPH compared to sarcoidosis patients without PH. (Alhamad et al., 2010; Baughman et al., 2007; Bourbonnais \& Samavati, 2008)

\section{Diagnosis}

Initial diagnosis of SAPH is based on a high index of suspicion. If pulmonary hypertension is clinically suspected in patients with sarcoidosis, a comprehensive series of diagnostic tests should be performed to confirm the diagnosis and to exclude other causes of pulmonary hypertension. A 2- dimensional echocardiography is an appropriate initial step to screen for $\mathrm{SAPH}$. Basic evaluation includes an electrocardiogram and laboratory tests, such as liver function tests, HIV testing, and markers of connective tissue disease. Ventilation-perfusion scan should be performed to rule out chronic thrombo-embolic pulmonary hypertension as an alternative explanation for pulmonary hypertension in these patients. Pulmonary function tests and tests of exercise capacity (such as the 6-minute-walk test or cardio-pulmonary exercise testing) are recommended to gauge baseline functional status of the SAPH patient.

The gold standard for diagnosis of SAPH is right heart catheterization (RHC), although there are no published guidelines for which patients should undergo RHC in this patient population. Given the high prevalence of pulmonary venous hypertension in patients with sarcoidosis, RHC is the only reliable diagnostic method to differentiate precapillary SAPH from postcapillary PVH.

We recommend that RHC be performed in all patients in whom SAPH is suspected, particularly if initiation of $\mathrm{PH}$ therapy is contemplated.

\subsection{Pulmonary Function Tests (PFTs)}

Compared to patients with sarcoidosis without $\mathrm{PH}$, patients who develop SAPH tend to demonstrate more pulmonary functional abnormalities and a more pronounced reduction in the diffusing capacity of the lung for carbon monoxide (DLCO). 
Forced vital capacity\% (FVC \%), forced expiratory volume in 1 second \% $\left(\mathrm{FEV}_{1} \%\right)$, midexpiratory flow (FEF $25-75)$, DLCO \%, and total lung capacity \% (TLC \%) have been reported to be significantly lower in patients with sarcoidosis and $\mathrm{PH}$ when compared with patients without PH. (Baughman et al., 2007; Bourbonnais \& Samavati, 2008; Handa et al., 2006; Sulica et al., 2005)

A very common finding in patients with $\mathrm{PH}$ is the presence of a disproportionate decrease in the diffusing capacity of the lung for carbon monoxide to the degree of pulmonary impairment and restriction, similar to PAH associated with scleroderma.

Decreases in DLCO \% below 50\% in the absence of radiographic pulmonary fibrosis and below $30 \%$ in patients with stage 4 CXR may signify the development of SAPH. (Sulica et al., 2005)

\subsection{Six Minute Walk Test (6MWT)}

The six minute walk test is an inexpensive, simple and reproducible test used to assess the functional status of patients with pulmonary, cardiac and pulmonary vascular disease. Six minute walk distance is reduced in the majority of patients with sarcoidosis, and this reduction is even more pronounced in patients with SAPH, when compared with sarcoidosis patients without PH. (Baughman et al., 2007, Bourbonnais \& Samavati, 2008) In a prospective study of 142 patients with sarcoidosis, the median 6MWD walked by SAPH patients was $280 \mathrm{~m}$, which was significantly lower compared to the median distance of 411 $\mathrm{m}$, walked by the patients without documented PH. (Baughman et al., 2007)

In addition, compared to patients without $\mathrm{PH}$, during a 6MWT, SAPH patients demonstrate more significant degree of oxygen desaturation, increased Borg Dyspnea Score and a lower distance saturation product (DSP, defined as the product between the distance walked and the lowest oxygen saturation achieved during the 6MWT). (Alhamad et al., 2010; Baughman et al., 2007; Bourbonnais \& Samavati, 2008) We recommend using the 6MWT at initial evaluation and periodically thereafter to monitor disease progression and response to therapy. However, it is important to recognize that, as opposed to patients with non-sarcoid pulmonary arterial hypertension (PAH), the clinical significance of the 6MWT results is more complex in SAPH patients. In these patients, other factors besides the presence of pulmonary vascular disease determine the $6 \mathrm{MWD}$, such as the degree of desaturation with exercise, lung functional parameters (FVC), and self reported respiratory health. (Baughman et al., 2007)

\subsection{Radiology}

As mentioned above, SAPH is more frequently encountered in patients with advanced radiographic stage and pulmonary fibrosis. The role of high resolution chest tomography (HRCT) in diagnosing SAPH is unclear. Compared to controls with sarcoidosis but no $\mathrm{PH}$, Nunes et al noted a more frequent occurrence of ground glass attenuation in patients with SAPH without fibrosis, and significantly higher frequency of septal lines in patients with SAPH and pulmonary fibrosis. Conversely, Handa et al were unable to correlate the presence of lymph node enlargement, lung opacities and thickening of bronchovascular bundles on HRCT with SAPH.

Thus, pending additional results, at this time it is reasonable to conclude that tomographic imaging studies are usually not helpful in predicting the presence of SAPH. (Handa et al., 2006; Nunes et al., 2006) 


\subsection{Echocardiography}

Transthoracic echocardiography (TTE) is a noninvasive screening method commonly used to test for the presence of pulmonary hypertension in sarcoidosis. It is also helpful to detect other cardiac abnormalities, such as left heart disease, presence of shunts or pericardial effusion, and particularly to evaluate the right heart, anatomically and functionally. Echocardiography may be used to estimate the right ventricular systolic pressure (RVSP) from the tricuspid regurgitation jet velocity. Arcasoy et al reviewed 374 patients with advanced lung disease and found that RVSP could be estimated in only $44 \%$ of the subjects due to the lack of tricuspid regurgitant jet in the majority of patients. (Arcasoy et al., 2003). For the patients in whom RVSP estimation was feasible, even though there was a good correlation with RHC-determined RVSP ( $r=0.69, \mathrm{p}<0.0001)$, the agreement was very poor. Notably, echocardiographically estimated RVSP differed from RHC measured RVSP by more than $10 \mathrm{mmHg}$ in $52 \%$ of cases. This study suggests that echocardiography may be a useful screening tool for pulmonary hypertension, but may not be accurate enough for quantification of pulmonary artery pressures. As such, echocardiography cannot substitute right heart catheterization for the accurate diagnosis of SAPH.

\subsection{Right Heart Catheterization (RHC)}

RHC is the gold standard for diagnosis of SAPH and for determining the degree of right ventricular dysfunction. In a single-center series, 53 patients with sarcoidosis and persistent dyspnea despite systemic immunosuppressive therapy underwent right heart catheterization. Notably, TTE was unable to detect RVSP in $30 \%$ of these patients and $24 \%$ of the patients with elevated RVSP on TTE had PCWP greater than $20 \mathrm{mmHg}$. This study underscores the importance of RHC in precisely identifying and categorizing $\mathrm{PH}$ in sarcoidosis. (Baughman et al., 2006) In a subsequent study, Baughman and colleagues performed RHC in 130 patients with sarcoidosis and unexplained dyspnea. They found that 70 patients $(53.8 \%)$ had $\mathrm{PH}$. Of these, 20 patients $(15.4 \%)$ had postcapillary, pulmonary venous hypertension ( $\mathrm{PH}$ with left ventricular disease), while 50 patients $(38.5 \%)$ had precapillary $\mathrm{PH}$, with normal left-sided filling pressures. Importantly, the presence of $\mathrm{PH}$ was associated with a higher risk for death; patients with precapillary $\mathrm{PH}$ and post-capillary $\mathrm{PH}$ had hazard ratios for death of 10.39 and 3.14 respectively. (Baughman et al., 2010) In conclusion, RHC is of paramount importance in the diagnosis of SAPH, not only for establishing an accurate diagnosis, evaluation of the right ventricular function and ruling out pulmonary venous hypertension, but also for valuable prognostic information.

\section{Treatment}

There are no specific therapies or treatment guidelines currently available for the management of $\mathrm{PH}$ in association with sarcoidosis. The current management of these patients is based on PAH therapeutic options and from observational studies.

\subsection{Corticosteroids and immunosuppressive therapy}

Corticosteroids and other immunosuppressive agents have been long used for the treatment of sarcoidosis, but they do not have an established role in the treatment of SAPH. Gluskowski et al used RHC to study 22 patients with pulmonary sarcoidosis and radiographic stage 2 and 3 disease that were treated with corticosteroids for 12 months. 
Radiological regression was seen in $91 \%$ of the patients. At baseline, $75 \%$ of the evaluated patients had elevated PAP with exercise and $16 \%$ had elevated PAP at rest. They found that half of the patients in each group had markedly reduced mPAP after treatment, but were unable to correlate corticosteroid treatment with changes in lung function, radiographic changes or mPAP decrease. (Gluskowski et al., 1990) A possible explanation for the lack of uniform hemodynamic response to corticosteroids in this study is that, in patients without a hemodynamic response, healing of the intravascular granulomas with fibrosis resulted in further narrowing of the pulmonary vessels and permanently increased pulmonary vascular resistance.

Nunes and colleagues found an even lower response to corticosteroids. They retrospectively studied 10 patients with SAPH treated with corticosteroids (oral prednisone 0.5 to 1 $\mathrm{mg} / \mathrm{kg} /$ day). These patients were evaluated at 3 and 6 months by Doppler echocardiography. In 3 of the 10 patients without evidence of pulmonary fibrosis they noted a decrease of more than $20 \%$ from baseline in RVSP by echocardiography. None of the patients with stage IV chest radiograph showed significant hemodynamic response to corticosteroids. (Nunes et al., 2006)

\subsection{Specific pulmonary hypertension therapy}

\subsubsection{Prostacyclins}

Intravenous epoprostenol is a prostaglandin with potent vasodilatory activity and inhibitory effect on platelet aggregation used for the treatment of PAH. Fisher et al evaluated the longterm effect of intravenous epoprostenol in 8 patients with moderate to severe $\mathrm{PH}$ (mean PVR $>1,176$ dynes $\mathrm{s} \mathrm{cm}^{-5}$ ) and WHO functional class III and IV symptoms. Majority of the patients showed evidence of pulmonary fibrosis, had restrictive physiology, decreased DLCO $\%$ and required supplemental oxygen. One of the 8 patients died from right heart failure prior to RHC. An acute vasodilator trial with intravenous epoprostenol was done in 7 of the 8 patients. Six of the 7 patients demonstrated acute vasoreactivity defined as a $25 \%$ or more decrease in the PVR in response to maximally tolerated dose of epoprostenol. These 6 patients were started on intravenous epoprostenol, but one died from cardiac arrest within hours of initiation. Five patients were followed for an average of 29 months while receiving intravenous epoprostenol (mean dose $55.6 \mathrm{ng} / \mathrm{kg} / \mathrm{min}$ ) and all showed functional improvement by 1 or $2 \mathrm{WHO}$ classes. This limited evidence suggests that PAH therapies may be beneficial for SAPH patients. (Fisher et al., 2006)

Inhaled iloprost, a prostacyclin analogue, has been administered as monotherapy to 22 patients with SAPH in an open label prospective study. Fifteen patients completed 16 weeks of iloprost administration, most common causes of discontinuation being cough and lack of compliance with 6-9 daily inhalations. Six patients showed hemodynamic improvement, with a more than $20 \%$ decrease in PVR. The $6 \mathrm{MWD}$ increased by more than $30 \mathrm{~m}$ in 3 patients. Quality of life, as measured by the Saint George Respiratory Questionnaire, also improved in 7 of the SAPH patients who completed the study. (Baughman et al., 2009)

\subsubsection{Phosphodiesterase inhibitors}

Selective inhibitors of cGMP-specific phosphodiesterase type 5 (PDE5) increase local NO in the pulmonary vasculature, thereby promoting vasodilation and are currently approved for 
the treatment of PAH. Only limited data is available for their use in SAPH. A small, single center retrospective study evaluated 25 patients with end-stage pulmonary sarcoidosis referred for transplantation. Twelve patients were treated with sildenafil for 1-12 months. Although there was no significant impact on the $6 \mathrm{MWD}$, a significant reduction in mPAP (average mPAP for the group decreased from $48 \mathrm{mmHg}$ to $39 \mathrm{mmHg}$ ), PVR (average PVR decreased from 10.7 to 5.6 Wood units), and an increase in the cardiac index from 2.3 to $2.9 \mathrm{~L} / \mathrm{min} / \mathrm{m}^{2}$ ) was noted. (Milman et al., 2008)

\subsubsection{Endothelin receptor blockers}

Endothelin-1 receptor blockers are used in PAH to reverse the endothelin system activation, which results in deleterious vasoconstriction and cardiac and vascular remodeling. Increased levels of endothelin-1 have been found in patients with sarcoidosis that have developed $\mathrm{PH}$, suggesting this might be a contributing mechanism to the development of SAPH. Published experience with endothelin receptor blockers in SAPH is limited to a few retrospective case series and one prospective study.

Baughman and colleagues evaluated 53 patients with sarcoidosis and persistent dyspnea and assessed their pulmonary pressures by RHC. Out of those, 5 patients with SAPH were treated with bosentan for 4 months, with a decrease in mPAP from $50 \mathrm{mmHg}$ to $35 \mathrm{mmHg}$ at follow-up. (Baughman et al., 2006; 2007)

We have reported a retrospective case series of 40 patients with SAPH who received bosentan and were followed for an average of 38 months. These patients demonstrated a significant reduction in their mean PAP and PVRI at follow-up RHC. $(\mathrm{P}=0.0048$ and $\mathrm{P}=0.017$, respectively). We also observed an increase in the 6MWD of $74.6 \mathrm{~m}(\mathrm{p}=0.0013)$ and less dyspnea. (Palmero \& Sulica, 2011)

Judson and colleagues enrolled $21 \mathrm{SAPH}$ patients in a proof of concept prospective study designed to investigate the effect of ambrisentan therapy over 6 months. Ten patients completed the study. There were no significant changes in the $6 \mathrm{MWD}$, gas exchanges or hemodynamic variables. However, there was a significant improvement in the WHO functional class and quality of life assessed by the Sarcoidosis Health Questionnaire. (Judson et al., 2011)

\subsubsection{Two-center experience}

Barnett et al evaluated 22 patients with SAPH treated with specific PAH therapeutic agents. These patients had moderate restrictive pulmonary dysfunction, severely decreased DLCO, radiologic stage IV disease (68\%), and a mean PAP of $46 \mathrm{~mm} \mathrm{Hg}$. Following treatment with different $\mathrm{PH}$ agents (sildenafil, bosentan, and intravenous epoprostenol), there were improvements in WHO functional class, 6MWD $(\mathrm{p}=0.032)$, mean PAP $(\mathrm{p}=0.008)$ and PVR $(p=0.011)$. Transplant free survival rates at 1 and 3 years were reported as $90 \%$ and $74 \%$, respectively, and no serious adverse events were attributed to the any of the drugs. (Barnett et al., 2009)

All these findings suggest a potential benefit from specific $\mathrm{PAH}$ therapeutic agents in selected patients with SAPH. However, large multicenter trials are required prior to recommending their use as standard of care in patients with SAPH. 


\section{Prognosis}

The development of $\mathrm{PH}$ reduces life expectancy in patients with sarcoidosis. Certain risk factors have been associated with worse outcomes and increased mortality. These factors include the presence of hypoxemia, mPAP above $35 \mathrm{mmHg}$, a cardiac index below $2 \mathrm{~L} / \mathrm{min} / \mathrm{m}^{2}$, and high right atrial pressure $\geq 15 \mathrm{Hg}$. (Arcasoy et al., 2001; Shorr et al., 2002)

\section{Conclusion}

$\mathrm{SAPH}$ is common in advanced sarcoidosis, although it can be found in earlier stages of the disease as well. Presence of SAPH is usually associated with increased or unexplained dyspnea, decreased functional status, increased oxygen requirements, and lower survival rates. When clinically suspected, appropriate diagnostic tests, particularly echocardiography and right heart catheterization, should be promptly performed. There is a variable and inconsistent response to the available therapeutic agents in small series of patients with SAPH. Further studies are necessary to better understand this disease and to define more effective therapeutic strategies.

\section{References}

Alhamad EH, Ahmad Shaik S, Idrees MM, et al. Outcome measures of the 6 minute walk test: relationships with physiologic and computed tomography findings in patients with sarcoidosis. Pulmonary Medicine 2010, 10: 42.

Arcasoy S, Christie J, Pochettino A, et al. Characteristics and outcomes of patients with sarcoidosis listed for lung transplantation. Chest 2001; 120:873.

Arcasoy S, Christie J, Ferrari V, et al. Echocardiographic assessment of pulmonary hypertension in patients with advanced lung disease. Am J Respir Crit Care Med 2003; 167: 735.

Badesch DB, Hunter CC, Gomez Sanchez Miguel Angel et al, Diagnosis and Assessment of Pulmonary Arterial Hypertension. J Am Coll Cardiol 2009; 54: S55-66.

Barnett C, Bonura E, Nathan S, et al. treatment of sarcoidosis-associated pulmonary hypertension. Chest 2009; 135:1455.

Baughman RP, Engel PJ, Meyer CA et al. Pulmonary hypertension in sarcoidosis. Sarcoidosis Vasc Diffuse Lung Dis 2006; 23:108.

Baughman R, Sparkman B, Lower E. Six-Minute Walk Test and Health Status Assessment in Sarcoidosis. Chest 2007; 132:207.

Baughman, RP. Pulmonary hypertension associated with sarcoidosis. Arthritis Research \& Therapy 2007; 9:S2.

Baughman RP, Judson MA, Lower EE, et al. Inhaled iloprost for sarcoidosis-associated pulmonary hypertension. Sarcoidosis Vasculitis and Diffuse Lung Diseases 2009; 26; 110-120.

Baughman RP, Engel PJ, Taylor L et al. Survival in Sarcoidosis-Associated Pulmonary Hypertension. The Importance of Hemodynamic Evaluation. Chest 2010 ; 138 (5) : 10781085.

Baughman RP, Culver DA, and Judson MA. A Concise Review of Pulmonary Sarcoidosis. Am J Respir Crit Care Med 183: 573-581, 2011

Bourbonnais JM and Samavati L. Clinical predictors of pulmonary hypertension in sarcoidosis. Eur Respir J 2008 ; 32 : 296-302.

Damuth T, Bower J, Cho K, et al. Major pulmonary artery stenosis causing pulmonary hypertension in sarcoidois. Chest 1980; 78:888. 
Fisher K, Serlin D, Wilson K et al. Sarcoidosis-associated pulmonary hypertension. Chest 2006; 130:1481.

Giaid A, Yanagisawa M, Langleben D, et al. Expression of endothelin-1 in the lung of patients with pulmonary hypertension. N Engl J Med 1993; 328:1732.

Gluskowski J, Hawrylkiewicz I, Zych D, et al. Effect of corticosteroid treatment on pulmonary haemodynamics in patients with sarcoidosis. Eur Respir J 1990; 3: 403.

Handa T, Nagai S, Miki S, et al. Incidence of pulmonary hypertension and its clinical relevance in patients with sarcoidosis. Chest 2006; 129:1246.

Judson MA, Kwon S, Highland KB, et al. The assessment of three health-related quality of life measurements assessed in an ambrisentan trial for sarcoidosis-associated pulmonary hypertension. Sarcoidosis Vasculitis and Diffuse Lung Diseases 2011; 28 (Suppl N:1); 20, E8.

Letizia C, Danese A, Reale M, et al. Plasma levels of endothelin-1 increase in patients with sarcoidosis and fall after disease remission. Panminerva Med 2001; 43:257.

Milman N, Burton C, Iversen M, et al. Pulmonary hypertension in end-stage pulmonary sarcoidosis: therapeutic effects of sildenafil?. J Heart Lung Transplant 2008; 27:329.

Nunes H, Humbert M, Capron F, et al. Pulmonary hypertension associated with sarcoidosis: mechanisms, haemodynamics and prognosis. Thorax 2006; 61:68.

Palmero V, Sulica R. Sarcoidosis-Associated Pulmonary Hypertension: Assessment and Management. Sem Resp Crit Care Med. 2010;31:494-500.

Palmero V, Sulica R. Bosentan for the Treatment of Sarcoidosis-Associated Pulmonary Hypertension. Am. J. Respir. Crit. Care Med., May 2011; 183: A5889.

Preston I, Klinger J, Landzberg M, et al. Vasoresponsiveness of Sarcoidosis-associated pulmonary hypertension. Chest 2001; 120:866.

Reichenberger F, Schauer J, Kellner k, et al. Different expression of endothelin in the bronchoalveolar lavage in patients with pulmonary Disease. Lung 2001; 179:163.

Rosen Y, Moon S, Huang C, et al. Granulomatous pulmonary angiitis in sarcoidosis. Arch Pathol Lab Med 1977; 101:170.

Shorr A, Davies D, Nathan S. Outcomes for patients with sarcoidosis awaiting lung transplantation. Chest 2002; 122:233.

Shorr AF, Helman DL, Davies DB, et al. Pulmonary hypertension in advanced sarcoidosis: epidemiology and clinical characteristics. Eur Respir J 2005; 25:783.

Simonneau G, Robbins IM, Beghetti M, et al. Clinical classification of pulmonary hypertension. J Am Coll Cardiol 2009; 54: S43-54.

Sofia M, Mormile M, Faraone $S$, et al: Endothelin-1 excretion in urine in active pulmonary sarcoidosis and in other interstitial lung diseases. Sarcoidosis 1995; 12:118.

Sterling R, Creager M. Nitric oxide and pulmonary hypertension. Coron Artery Dis 1999; 10:287.

Sulica R, Teirstein AS, Kakarla S, et al. Distinctive clinical, radiographic and functional characteristic of patients with sarcoidosis-related pulmonary hypertension. Chest 2005; 128:1483.

Takemura T, Matsui Y, Oritsu M, et al. Pulmonary vascular involvement in sarcoidosis: granulomatous angiitis and microangiopathy in transbronchial lung biopsies. Virchows Arch A Pathol Anat Histopathol 1991; 418:281.

Takemura T, Matsui Y, Saiki S, et al. Pulmonary vascular involvement in sarcoidosis: a report of 40 autopsy cases. Hum Pathol 1992; 23:1216.

Terashita K, Kato S, Sata M, et al. Increased endotelin-1 levels in BAL fluid in patients with pulmonary sarcoidosis. Respirology $2006 ; 11: 145-151$

Wescott J, DeGraff A. Sarcoidosis, hilar adenopathy and pulmonary artery narrowing. Radiology 1973; 108:585. 


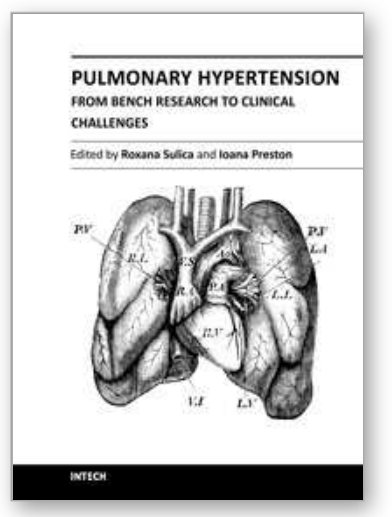

\section{Pulmonary Hypertension - From Bench Research to Clinical Challenges}

Edited by Dr. Roxana Sulica

ISBN 978-953-307-835-9

Hard cover, 326 pages

Publisher InTech

Published online 09, December, 2011

Published in print edition December, 2011

The textbook "Pulmonary Hypertension - From Bench Research to Clinical Challenges" addresses the following topics: structure and function of the normal pulmonary vasculature; disregulated cellular pathways seen in experimental and human pulmonary hypertension; clinical aspects of pulmonary hypertension in general; presentation of several specific forms of pulmonary hypertension, and management of pulmonary hypertension in special circumstances. The textbook is unique in that it combines pulmonary and cardiac physiology and pathophysiology with clinical aspects of the disease. First two sections are reserved for the basic knowledge and the recent discoveries related to structure and cellular function of the pulmonary vasculature. The chapters also describe disregulated pathways known to be affected in pulmonary hypertension. A special section deals with the effects of hypoxia on the pulmonary vasculature and the myocardium. Other three sections introduce the methods of evaluating pulmonary hypertension to the reader. The chapters present several forms of pulmonary hypertension which are particularly challenging in clinical practice (such as pulmonary arterial hypertension associated with systemic sclerosis), and lastly, they address special considerations regarding management of pulmonary hypertension in certain clinical scenarios such as pulmonary hypertension in the critically ill.

\section{How to reference}

In order to correctly reference this scholarly work, feel free to copy and paste the following:

Veronica Palmero, Phillip Factor and Roxana Sulica (2011). Sarcoidosis Associated Pulmonary Hypertension, Pulmonary Hypertension - From Bench Research to Clinical Challenges, Dr. Roxana Sulica (Ed.), ISBN: 978953-307-835-9, InTech, Available from: http://www.intechopen.com/books/pulmonary-hypertension-frombench-research-to-clinical-challenges/sarcoidosis-associated-pulmonary-hypertension

\section{INTECH}

open science | open minds

\author{
InTech Europe \\ University Campus STeP Ri \\ Slavka Krautzeka 83/A \\ 51000 Rijeka, Croatia \\ Phone: +385 (51) 770447 \\ Fax: +385 (51) 686166 \\ www.intechopen.com
}

\author{
InTech China \\ Unit 405, Office Block, Hotel Equatorial Shanghai \\ No.65, Yan An Road (West), Shanghai, 200040, China \\ 中国上海市延安西路65号上海国际贵都大饭店办公楼 405 单元 \\ Phone: +86-21-62489820 \\ Fax: +86-21-62489821
}


(C) 2011 The Author(s). Licensee IntechOpen. This is an open access article distributed under the terms of the Creative Commons Attribution 3.0 License, which permits unrestricted use, distribution, and reproduction in any medium, provided the original work is properly cited. 